\title{
Liver Transplantation and Surgery in the Management of Hepatocellular Carcinoma
}

\author{
Massimo Malagó ${ }^{1 *}$, Dimitri Aristotle Raptis ${ }^{2}$, Charles J. Imber ${ }^{2}$, Giovanni Dalla Serra ${ }^{3}$, \\ Oskar Kornasiewicz ${ }^{4}$
}

'University College London, London, United Kingdom

${ }^{2}$ Department of HPB Surgery and Liver Transplantation, Royal Free London,

NHS Foundation Trust, United Kingdom

${ }^{3}$ Surgical Operative Unit, Villa Igea Hospital, Ancona, Italy

${ }^{4}$ Departmentof General, Transplant and Liver Surgery, Medical University of Warsaw, Poland

\section{ABSTRACT}

Hepatocellular carcinoma (HCC) is the second cause of cancer-related deaths worldwide constitutes a major global health problem. Although our understanding of the molecular pathogenesis of hepatocellular HCC has improved, detected driver mutations are not yet sufficiently reliable therapeutic targets. The modern management of HCC is conceived only within multidisciplinary schemes. Current systemic therapies are not curative and surgical treatments remain the only potential curative treatments for selected patients with early stage HCC. The role of parenchyma sparing liver resection for appropriately selected patients may vary in different environments according to organ availability,and liver resection remains a reliable curative method for HCC. Meta-analyses have shown that clinical outcomes of surgical resection are superior to ablative treatments in early stage HCC. Liver transplantation is still the standard treatment for patients with early stage HCC. Lately the Milan Criteria are considered too restrictive, still recognizing that the extension of transplantation criteria beyond Milano criteria increases the risk of HCC recurrence. Resection, ablation, transarterial embolization and trans-arterial radiation are commonly applied to bridge patients to transplant. Despite enormous advances in the therapy of HCC treatments at present, there are currently no genuine breakthroughs for patients with HCC. The Clinical Trials Planning Meeting (CTPM) in HCC convened by the American Society of Clinical Oncology identified the key knowledge gaps in HCC and define clinical research priorities. Major improvements in survival provided by multimodal treatments witnessed in other cancers are not yet available for HCC because effective systemic treatment modalities for HCC are still lacking. Integration of bio-genetic tumor information into current tested treatment schemes and truly multimodal surgery-based treatment schemes will enable the recruitment of less selected patients in larger numbers to curative treatments.

Key words: Hepatocellular carcinoma, liver, cancer, surgery, management, future

\author{
*Corresponding author: \\ Professor Massimo Malago, MD, PhD \\ Department of HPB Surgery and Liver \\ Transplantation \\ The London Digestive Centre \\ 41 Welbeck Street, London W1G 8DU \\ E-mail: m.malago@ucl.ac.uk
}

\section{INTRODUCTION}

Hepatocellular carcinoma ( $\mathrm{HCC}$ ) is a major global health problem and the 
second cause of cancer-related deaths worldwide, with an estimated 850,000 new cases annually. Unfortunately, the prognosis for liver cancer is very poor, with a ratio of mortality to incidence of 0.95 , with the rare cure still achieved only by surgical modalities. In more than $80 \%$ of patients, HCC develops in cirrhotic liver $(1,2)$. This diversity in etiology and natural history results in high $\mathrm{HCC}$ heterogeneity, raising major challenges in clinical management (2). Primary prevention of HCC by HBV vaccination has been reported in many studies. Additionally, the HCC risk decreases after the first 5 years of antiviral therapy in chronic hepatitis $B$ patients (3). Similarly, chronic hepatitis $C$ patients receiving direct antiviral agent (DAA) who have achieved sustained virologic responses (SVR) were found to be associated with a decrease in HCC risk (4-6).

The modern management of HCC is conceived only within multidisciplinary schemes. Surgical treatment of patients with HCC shall be restricted to centers with availability of all treatment methods, including liver transplantation, or at least to centers with organized network connections to twinning programs offering multidisciplinary holistic treatment of HCC as well as of liver diseases. Liver resection, liver ablation therapies, and liver transplantation remain the mainstay of surgical interventional therapy, in combination with medical/radiological methods.

Modern diagnosis and treatment of HCC occur in a multimodal approach. Our understanding of the molecular pathogenesis of HCC has improved significantly in the last decade. Recent advances have led to identification of critical driver mutations, however most mutations are not yet a target for pharmacologic or immunologic therapies. Surveillance programs can detect early stage HCCs that are amenable to curative treatments such as surgical resection, liver transplantation, and local ablation. Treatment, and particularly cure of HCC,is still a major challenge; the application of surgical therapies iscurrently the only curative chances in a very selected category of patients.

\section{Current management of HCC}

Current therapeutic strategies for HCC are based on number of tumors, size, vascular invasion, functional reserve of the liver and clinical performance status $(2,6$, 7), variables all included in the Barcelona Clinic Liver Cancer classification (BCLC). This treatment algorithm has been a standard of care for long years and has been constantly updated. Lately, however, BCLC has been criticized and updated by other treatment algorithms and guidelines from both western and eastern societies and groups (8-11).

Surveillance programs currently detect early-stage tumors (single $>2 \mathrm{~cm}$, or 3 or fewer nodules $<3 \mathrm{~cm}$ ) in patients that are candidates for potentially curative therapies (local ablation, liver resection, or transplantation) with 5 - year survival rates of $50 \%-70 \%(2,7,12)$. The 5-year recurrence rates after $\mathrm{HCC}$ resection was shown to be up to $70 \%$, or even higher after percutaneous ablation, making liver transplantation the best possible option in very selected cases, whenever sufficient liver grafts are available. Post-transplant recurrence rates reach approximately $10 \%$ and liver transplantation (LT) offers the additional advantage of eliminating the underlying liver disease $(2,12)$.

HCC develops mostly in cirrhotic liver $(1,2)$, particularly in the background of viral hepatitis and non-alcoholic steatohepatitis (NASH). This diversity in etiology and natural history results in high HCC heterogeneity, raising major challenges in clinical management (2). Primary prevention HCC by hepatitis $B$ virus (HBV) vaccination has been reported in many studies. The $\mathrm{HCC}$ risk decreases after the first 5 years of antiviral therapy in chronic hepatitis B patients (3). Similarly, chronic hepatitis $C$ patients receiving direct antiviral agent (DAA) who have achieved sustained virologic responses (SVR) were found to be associated with a decrease in HCC risk (4-6).

\section{Tumor ablation and liver resection strategies}

\section{Ablation versus resection/transplantation}

There has been a hot debate in the scientific community whether surgical approaches or interventional therapies for very early or early stage HCC patients should be preferred. Recently, a meta-analysis published by Ni JY et al. including approximately 21,000 patients demonstrated that clinical outcomes of surgical resection were superior to ablative treatments in early stage HCC (13). These findings were confirmed by another meta-analysis published by Xu et al. that showed that surgical procedures provide significantly improved survival benefits in patients with early stage HCC when compared to patients underwent ablative treatments $(14,15)$.

A meta- analysis of available studies including 8500 HCC patients has demonstrated that in very early HCC patients with Child - Pugh A, Radiofrequency Ablation (RFA) provides similar survival to resection (16). However, for single larger HCCs $(3-5 \mathrm{~cm})$ resection provided better survival and was more cost effective than RFA (16). In "small HCC" - less than $2 \mathrm{~cm}$ with 2 the 
choice of tumor ablation (percutaneous ethanol injection (PEI) or RFA) is dictated by operability of the patient, liver reserve, portal vein tumor invasion.

\section{Liver resection for $\mathrm{HCC}$}

In the current environment of organ shortage, liver resection remains a mainstay of treatment, albeit still used in a very selected population. Patients with HCC without macrovascular invasion, with preserved liver function (Child - Pugh class A with bilirubin of $<1$ $\mathrm{mg} / \mathrm{dl}$ ) and no/moderate portal hypertension are considered to be optimal candidates for surgical resection $(2,17,18)$. Low rates of $(<2 \%)$ perioperative mortality with approximately $30 \%-50 \%$ disease free survival at 5 years have been reported by several studies in these patients. A drawback of liver resection is $\mathrm{HCC}$ recurrence in the remnant liver, a common event and frequent cause of mortality.Limited resection is preferred when possible, to spare uninvolved liver parenchyma still removing satellite nodules that result from local vascular invasion (2). Indeed, liver resection for HCC has evolved to a standard of "parenchyma sparing policy", avoiding major resections whenever possible, because of important morbidity and mortality related to post-hepatectomy liver failure (PHLF). The tendency of HCC to propagate via portal vein invasion and the propensity to build peri-HCC satellite metastases, born in micro-portal vein tumor thrombi, requires the hepatectomy to follow segmental or subsegmental portal vein patterns, as "portal vein directed". The evaluation of liver reserve in candidates for liver resection in cirrhosis is a key feature to achieve optimal short term post-hepatectomy results. While in the western world bilirubin, Child-Pugh classification and portal vein pressure have been for years the principal variables for safe liver resection, with the late addition of, in the eastern surgical centers clinical variables have been compounded with Indocyanine Green Test (ICG) to tailor and refine the selection of candidates who can survive liver resection with sufficient liver reserve. The protocol introduced by Makuuchi (19) is now widely adopted in the eastern world, and selected centers use it in the western world as the best selective method to achieve good short term results.

When a major resection is required, preliminary portal vein embolization or lobar radioembolization can be performed in some centers to induce future liver remnant growth and avoid liver insufficiency (2). The failure to produce significant growth is a contraindication to the hepatectomy.

A meta-analysis showed that preoperative strate- gies are effective to increase liver volume in order to achieve resectability of HCC. Transarterial chemoembolization (TACE) and PVE is as safe as PVE providing higher overall survival. ALPPS (Associating Liver Partition and Portal vein Ligation for Staged hepatectomy) needs to be used with caution because of risks of major complications and ultimately mortality (20). Furthermore, radioembolization seems to present similar resection rates and overall survival as with TACE and PVE, but with higher rate of postoperative morbidity (21).

There has been great controversy on the utility of portal hypertension as parameter in the selection of liver resection candidates. A study published by Ohkubo et al. from Japan, demonstrated that portal hypertension did not negatively affect survival rates in patients who underwent liver resection for hepatocellular carcinoma (22). In the study, between 2001 and 2015 , the researchers analyzed the complications and survival outcomes of patients who underwent liver resection for HCC. In one group, 695 patients had neither portal hypertension nor multiple tumors, whereas a second group of 197 patients had both. Patients with portal hypertension and multiple tumors had significantly more postoperative complications than those in the first group ( $47 \%$ vs. $36 \%$ ) and significantly longer postoperative length of hospital stay (median, 13 vs. 12. It was concluded that liver resection is a safe surgical approach for patients with HCC and portal hypertension, but recurrence rate of HCC in patients with portal hypertension and multiple tumors were significantly higher. Therefore an optimal treatment for such patients meeting Milan criteria is liver transplantation (22).

At the Royal Free Hospital in London,liver resection candidates were selected using a combination of the Makuuchi criteria and hepatic vein-portal venous gradient (HVPG) screening for portal hypertension. Fifty-two hepatectomies were performed from 2010 to 2014 , achieving an R0 resection was in $92 \%$ of cases, post-operative 30 -day mortality was $1 \%$ and the overall survival at 5 years $68 \%$, whereas the 5 -year disease free survival $58 \%$. The presence of portal hypertensionand portal vein invasion correlated with outcomes in terms of morbidity and survival respectively, while R1 resection, multiple tumors and major hepatectomies correlated with decreased disease-free survival. Laparoscopic liver resection for HCC has become a mainstay method of liver resection, particularly for limited resections in peripheral segments (23).

Recently the Albumin-Bilirubin (ALBI)score emerged a reliable screening method predicting short term 
outcomes also for liver resections $(24,25)$. The Albumin-Indocyanine Green Evaluation (ALICE) score has instead been developed as useful criteria for prediction of successful long-term outcome after liver resections $(24,25)$. Thus, surgical resection may offer a curative option for HCC with a very low postoperative mortality and current selection criteria may be expanded in well-designed clinical trials.

\section{Liver transplantation}

\section{Staging}

Liver transplantation has become a standard treatment for patients with early stage $\operatorname{HCC}(2,12)$. Liver transplantation treats both the HCC and the underlying liver disease, usually liver cirrhosis, the main risk factor for developing HCC; therefore, the selected patients receiving transplants have the highest chance of cure among all other treatments (12). Liver transplantation for HCC in the background of cirrhosis is stil the best treatment option for patients with early stage tumors, with 5 -year overall survival exceeding $70 \%$ in appropriately selected cases. The Milan criteria are the most common criteria and a cornerstone to select HCC patients for transplantation. Novel surgical approaches have been developed to increase the pool of organs for liver transplantation such as living donor liver transplantation, donation after circulatory death and split livers.

Transplantation provides a 5-year survival rate of $70 \%$ with a recurrence rate of approximately $10 \%$ (2). Transplantation has the appeal of removing unrecognized intrahepatic metastasis and essentially eliminating the risk of de novo tumor development (12). The criteria for determining whether a patient with $\mathrm{HCC}$ is eligible for liver transplantation are very heterogeneous worldwide (12). However, the Milan criteria defined by Mazzaferro in 1996 remain the most common criteria to select HCC patients for liver transplantation (26). Since then, the Milan Criteria (single nodule $<5 \mathrm{~cm}$ or $<3$ nodules $<3 \mathrm{~cm}$ in size)became the gold standard and best predictor of post-transplant outcome and of cost-effective transplantation $(18,27,28)$. Lately the Milan Criteria are considered too restrictive, still recognizing that the extension of transplantation criteria beyond Milano criteriaincreases the risk of HCC recurrence. Starting with the University of California San Francisco (UCSF), many other criteria such as up-to-7, Total Tumor Volume (TTV) ,the Extended Toronto Criteria (ETC) and Kyoto Criteria have been proposed over time to predict the outcomes for $\operatorname{HCC}(2,7,17,18)$. Among extended criteria, UCSF and ETC have been validated in several studies. Most of these criteria have demonstrated the same survival results, but expanding the indications for liver transplantation has been associated with an increased HCC recurrence $(2,17)$.

\section{Host and tumor biologyas features of HCC treatment}

In patients with decompensated HCV cirrhosis direct acting antiviral (DAA) therapy is associated with improved hepatic function $(4,5)$. A recent multicenter European study showed that DAAs could reverse liver function and favored the inactivation and delisting of about one patient out-of-three and one patient out-of-five in 60 weeks, respectively (4). In this study, patients with lower MELD scores had a higher chance to be delisted. Briefly, using DAAs in daily practice will further increase organ availability for HCC patients in the near future, as a significant number of patients with decompensated cancer-free HCV cirrhosis will probably be inactivated and delisted within 1 year (4). Delisted patients may then become candidates for liver resection, or other therapies.

The use of only tumor size and number is not the optimal approach to select HCC patients for liver transplantation. More detailed information about HCC biology is needed. Hepatocellular carcinomas exhibit a diversity of molecular phenotypes, raising major challenges in clinical management $(2,7,29)$. Hepatocellular carcinomas develop from diverse etiological background and display remarkable biological and clinical heterogeneity $(29,30)$. The appreciation of the molecular pathogenesis of HCC has improved significantly in the last decade by understanding the landscape of mutations and critical pathways involved in the development and progression of HCC. Genomic analyses have provided a vast panorama of the main drivers responsible for tumor initiation and progression $(29,30)$. Each HCC has average of 40 genomic aberrations, among which few are considered drivers (2). At present, HCCs are divided into two molecular classes: proliferative and non-proliferative HCCs. Proliferative HCCs are more aggressive and less differentiated and are frequently associated with high serum alfa-fetoprotein (AFP) levels, p53 mutations and poor prognosis. Furthermore, proliferative HCCs may display transforming growth factor beta (TGF-B), MET, AKT and / or the progenitor cell phenotype $(2,7,29,30)$. By contrast, the non-proliferative subgroup appears to be heterogeneous with less - certain biologic significance (30).When available, this information may help choose 
the appropriate surgical management of these patients (e.g. Ablation vs. surgery vs. transplantation) in welldesigned clinical trials.

In this context, Mazzaferro has proposed a new paradigm in the management of LT in HCC patients: an Adaptive Approach (27), highlighting the significance of biological profiles and behaviors of HCC. This system applies only to early and intermediate stage HCC in compensated cirrhosis (BCLC - A and BCLC - B). Exclusion criteria are vascular invasion, extrahepatic metastasis and comorbidity. In principle, any HCC developing in compensated cirrhosis is considered as transplantable tumor (TT). Morphological criteria should be integrated with pathologic/biologic information. The best available option (i.e. monotherapy or combination therapy) should be determined through multidisciplinary discussion. A minimal observation period after the therapy is mandatory. The minimal expected survival for patients receiving LT under these conditions should be increased from the conventional limit of $50 \%$ at 5 years to $60 \%$ or higher (27).

Live Donor liver transplantation for HCC (31) has proven to be a valid tool both in the Eastern World with scarce deceased organ donor resources and in the Western World. In experienced centres applying careful candidate selection criteria excellent results may reached (32). This technique offers a particular timing advantage and could be considered more frequently.

\section{Bridging strategies for transplantation}

Patient drop-out on the waiting list because of HCC progression is a significant issue, giving relevance to strategies and therapies of patients waiting for LT. HCC progression on the waiting list may impair intention-totreat outcomes (2). Resection, ablation, trans-arterial embolization and trans-arterial radiation are commonly applied to bridge patients to transplant (2). Excessive priority for HCC compared to non-tumor indications can result in increased post-operative HCC recurrence (2, 12). In this context, several studies have demonstrated that AFP has significant prognostic potential. In general, HCC patients on the waiting list with baseline serum level of AFP $>200 \mathrm{ng} / \mathrm{ml}$ show significantly worse outcomes, although the most significant adverse determinant is the steady increase of AFP $>\mathrm{ng} / \mathrm{mL} /$ month. The AFP cut-off of 300,400 and $1000 \mathrm{ng} / \mathrm{mL}$ have been suggested as delisting indication $(30,33,34)$.

At the Royal Free Hospital in London, liver transplantation (OLT) candidates were selected strictly adhering to the Milano criteria, as customary in the UK. We reviewed the results of a study period of 3 years, from Jan 2013 to Dec 2015. A total of 48 consecutive cases without exclusions underwent OLT. There were 7 females (15\%) and 41 males for a $M / F$ ratio of 4.6 ; median age was 58 years (IQR 54-60). The main modality of downstaging used prior to OLT was TACE, in 32 cases (74\%). The minimum follow-up time was 2 years with a median of 39 months. Twelve patients received DCD grafts; all other patients received full DBD grafts. No patient required re-transplantation. Overall 1 and 3 year survival was 89.6 and $85 \%$, respectively. Disease free 1 and 3 year survival was 95.1 and $89.7 \%$, and graft 1 and 3 year survival was 89.6 and $83.0 \%$, respectively.

\section{The future}

The Clinical Trials Planning Meeting (CTPM) in HCC was convened by the US National Cancer Institute's Gastrointestinal Cancer Steering Committee and the American Society of Clinical Oncology to identify the key knowledge gaps in HCC and define clinical research priorities (35). These included, among others, conducting prospective trials of combined modalities, including TACE plus ablation and TACE plus systemic therapy, evaluating the outcome of regional therapy versus systemic therapy in patients with lymph node positive or intrahepatic metastatic disease, conducting comparison trials of TACE, drugeluting bead (DEB), and Y90-labeled microspheres, defining the role of TACE in liver transplantation and conducting prospective trials of regional therapy options. As for local therapy, there is a need for formally evaluating the outcome of adjuvant systemic therapy following resection or ablation, conducting a trial of adjuvant chemotherapy following intrahepatic therapy, DEB, or Y90-labeled microspheres and comparing modalities, such as hepatic resection versus ablation. The expectation of resolutive potential of internal radiation therapies has been unfortunately wasted in poorly designed randomized studies (36). For liver transplantation, the research questions should focus on weather adjuvant therapy may improve post-LT outcome in patients at high risk for recurrence (34).

Apart from primary prevention strategies and truly effective systemic treatments, the future of the management of HCC lies on surgery and liver transplantation combined with adjuvant therapy tailored according to the genetic profiling and stratification of the HCC including targeted immunotherapy. Organ and graft availability can be improved by novel approaches to organ procurement as extracorporeal machine perfusion (37), or Normothermic Regional 
Perfusion for expanded criteria grafts and donors (38). With regards to living donor transplantation, refining and expanding the novel RAPID procedure for patients with HCC inliver cirrhosis may be the future in highly selected patients with favored tumor biology and moderate portal hypertension. The novel RAPID procedure concept was developed for partial liver transplantation in nonresectable colorectal liver metastases with very small donor liver grafts (39). At the time of transplantation, segments 2 and 3 , or the left hemiliver, are resected in the recipient and orthotopically replaced by a Small-For-Size left (S2-3-4) or left lateral (S2-3) grafts (Deniz Article). Portal inflow is modulated by redirecting the portal flow to the graft. Indeed, a major difficulty of this approach in cirrhotic patients is the correct management of portal hypertension and SFS Syndrome. A second-stage hepatectomy, similarly to ALPPS, is performed as soon as the graft has regenerated to a sufficient volume and function. This represents a new procedure that needs validation, a possible strategy to increase the availability of liver transplantation to selected patients with nonresectable hepatocellular carcinoma.

The never-ending issue whether transplant is more effective than liver resection for "small HCC" has not yet been resolved. Organ shortage constitutes a major obstacle to appropriate randomized studies and even more for the eventual application of LT to all early HCC. Attempts of integration of biologic markers into clinical algorithms for better calculation of prognosis have been already positively tested. This modality may be a way forward in the optimal stratification of patients for treatment of HCC, and other cancers (40).

\section{CONCLUSION}

Although our understanding of the molecular pathogenesis of HCC has improved significantly and many HCC mutations have been identified, detected driver mutations are not yet sufficiently reliable therapy targets. Current systemic therapies are not curative and surgical treatments remain the only potential curative treatments for selected patients with early stage HCC. Hence, the institution of early HCC detection and surveillance programs is an essential tool to decrease overall global/national mortality from HCC. The role of liver resection for appropriately selected patients may vary in different environments according to organ availability, so LR remains a reliable curative method for HCC. Liver transplantation is still the standard treatment for patients with early stage HCC.
Surgical therapies remain the most effective treatments when applied to selected patients in early stages of the disease. Despite enormous advances in the therapy of HCC treatments at present, there are no real breakthroughs for patients with HCC. Indeed, and very unfortunately the major improvements in survival provided by multimodal treatments witnessed for other cancers are not yet available for $\mathrm{HCC}$ because effective systemic treatment modalities for HCC are still lacking. Integration of bio-genetic tumor information into current tested treatment schemes and the advent of truly multimodal surgerybased treatment schemes willenable the recruitment of more patients into curative treatments.

\section{Funding}

This work did not receive any funding; no other disclosure.

\section{Ethics of approval}

Ethical approval is not necessary.

\section{REFERENCES}

1. Akkiz H, Carr BI, Kendal Yalçın K, Guerra V, Kuran S, Altıntaș E, et al. Characteristics of Hepatocellular Carcinoma Aggressiveness Factors in Turkish Patients. Oncology. 2018;94(2):116-124. Epub 2017 Dec 6.

2. Bruix J, Gores GJ, Mazzaferro V. Hepatocellular carcinoma: clinical frontiers and perspectives. Gut. 2014;63(5):844-55

3. Papatheodoridis GV, Idilman R, Dalekos GN, Buti M, Chi H, van Boemmel $F$, et al. The risk of hepatocellular carcinoma decreases after the first 5 years of entecavir or tenofovir in Caucasians with chronic hepatitis B. Hepatology. 2017;66(5):1444-1453

4. Belli LS, Berenguer M, Cortesi PA, Strazzabosco M, Rockenschaub SR, Martini S, et al. Delisting of liver transplant candidates with chronic hepatitis C after viral eradication: A European study. J Hepatol. 2016;65(3):524-31.

5. Chhatwal J, Samur S, Kues B, Ayer T, Roberts MS, Kanwal F, et al. Optimal timing of hepatitis $C$ treatment for patients on the liver transplant waiting list. Hepatology. 2017;65(3):777-788.

6. Verna EC. The dynamic landscape of liver transplant in the era of effective hepatitis C virus therapy. Hepatology. 2017;65(3):763-766.

7. Llovet JM, Zucman-Rossi J, Pikarsky E, Sangro B, Schwartz M, Sherman M. Hepatocellular carcinoma. Nat Rev Dis Primers. 2016; 2:16018.

8. European Association for the Study of the L.R. European Organisation For, and C. Treatment Of, EASL-EORTC clinical practice guidelines: management of hepatocellular carcinoma. J Hepatol, 2012;56(4):908-43.

9. European Association for the Study of the Liver. Electronic address, e.e.e. and L. European Association for the Study of the, EASL Clinical Practice Guidelines: Management of hepatocellular carcinoma. J Hepatol. 2018;69(1):182-236.

10. Omata M, Lesmana LA, Tateishi R, Chen PJ, Lin SM, Yoshida H, et al. Asian Pacific Association for the Study of the Liver consensus recommendations on hepatocellular carcinoma. Hepatol Int. 2010; 4(2):439-74.

11. Kudo M, Izumi N, Kokudo N, Matsui O, Sakamoto M, Nakashima O, 
et al. Management of hepatocellular carcinoma in Japan: Consensus-Based Clinical Practice Guidelines proposed by the Japan Society of Hepatology (JSH) 2010 updated version. Dig Dis. 2011;29(3):339-64.

12. Sapisochin G, Bruix J. Liver transplantation for hepatocellular carcinoma: outcomes and novel surgical approaches. Nat Rev Gastroenterol Hepatol. 2017;14(4):203-217.

13. Ni JY, Xu L-f, Sun H-I, Zhou J-X, Chen Y-t, Luo J-h. Percutaneous ablation therapy versus surgical resection in the treatment for earlystage hepatocellular carcinoma: a meta-analysis of 21,494 patients J Cancer Res Clin Oncol. 2013; 139(12):2021-33

14. Slotta JE, Kollmar O, Ellenrieder V, Ghadimi BM, Homayounfar K. Hepatocellular carcinoma: Surgeon's view on latest findings and future perspectives. World J Hepatol. 2015;7(9):1168-83.

15. Xu G, Qi FZ, Zhang JH, Cheng GF, Cai Y, Miao Y. Meta-analysis of surgical resection and radiofrequency ablation for early hepatocellular carcinoma. World J Surg Oncol. 2012;10:163.

16. Cucchetti A, Piscaglia F, Cescon M, Colecchia A, Ercolani G, Bolond $L$, et al. Cost-effectiveness of hepatic resection versus percutaneous radiofrequency ablation for early hepatocellular carcinoma. J Hepatol. 2013;59(2):300-7

17. Clavien PA, Lesurtel M, Bossuyt PMM, Gores GJ, Langer B, Perrie $A$, et al. Recommendations for liver transplantation for hepatocellular carcinoma: an international consensus conference report. Lancet Oncol. 2012;13(1):e11-22. Epub 2011 Oct 31.

18. Sapisochin G, Goldaracena N, Laurence JM, Dib M, Barbas A, Ghanekar A, et al. The extended Toronto criteria for liver transplantation in patients with hepatocellular carcinoma: A prospective validation study. Hepatology. 2016;64(6):2077-2088.

19. Imamura H, Sano K, Sugawara Y, Kokudo N, Makuuchi M Assessment of hepatic reserve for indication of hepatic resection decision tree incorporating indocyanine green test. J Hepatobiliary Pancreat Surg. 2005;12(1):16-22.

20. Donati M, Basile F, Oldhafer KJ. Present status and future perspectives of ALPPS (associating liver partition and portal vein ligation for staged hepatectomy). Future Oncol. 2015;11(16):2255-8.

21. Tustumi F, Ernani L, Coelho FF, Bernardo WM, Junior SS, Kruger JAP, et al. Preoperative strategies to improve resectability for hepatocellular carcinoma: a systematic review and meta-analysis HPB (Oxford). 2018;20(12):1109-1118.

22. Ohkubo T, Midorikawa $\mathrm{Y}$, Nakayama H, Moriguchi M, Aramaki O, Yamazaki S, et al. Liver resection of hepatocellular carcinoma in patients with portal hypertension and multiple tumors. Hepatol Res. 2018;48(6):433-441.

23. Wakabayashi G, Cherqui D, Geller DA, Buell JF, Kaneko H, Han HS, et al. Recommendations for laparoscopic liver resection: a report from the second international consensus conference held in Morioka. Ann Surg. 2015;261(4):619-29.

24. Kokudo T, Hasegawa K, Amikura K, Uldry E, Shirata C, Yamaguchi T, et al. Assessment of Preoperative Liver Function in Patients with Hepatocellular Carcinoma - The Albumin-Indocyanine Green Evaluation (ALICE) Grade. PLoS One. 2016;11(7):e0159530.

25. Johnson PJ, Berhane S, Kagebayashi C, Satomura S, Teng M, Reeves $\mathrm{HL}$, et al. Assessment of liver function in patients with hepatocellular carcinoma: a new evidence-based approach-the ALB grade. J Clin Oncol. 2015;33(6):550-8. Epub 2014 Dec 15

26. Mazzaferro V, Regalia E, Doci R, Andreola S, Pulvirenti A, Bozzetti F, et al. Liver transplantation for the treatment of small hepatocellular carcinomas in patients with cirrhosis. N Engl J Med. 1996; 334(11):693-9.

27. Mazzaferro V. Squaring the circle of selection and allocation in liver transplantation for HCC: An adaptive approach. Hepatology. 2016; 63(5):1707-17.

28. Mazzaferro V, Bhoori S, Sposito C, Bongini M, Langer M, Miceli R, et al. Milan criteria in liver transplantation for hepatocellular carcinoma: an evidence-based analysis of 15 years of experience. Liver Transpl. 2011;17 Suppl 2:S44-57.

29. Zucman-Rossi J, Villanueva A, Nault JC, Llovet JM. Genetic Landscape and Biomarkers of Hepatocellular Carcinoma. Gastroenterology. 2015;149(5):1226-1239.e4.

30. Desert R, Rohart F, Canal F, Sicard M, Desille M, Renaud S, et al. Human hepatocellular carcinomas with a periportal phenotype have the lowest potential for early recurrence after curative resection. Hepatology. 2017;66(5):1502-1518.

31. Malagó M, Sotiropoulos GC, Nadalin S, Valentin-Gamazo C, Paul A, Lang $\mathrm{H}$, et al. Living donor liver transplantation for hepatocellular carcinoma: a single-center preliminary report. Liver Transpl. 2006; 12(6):934-40

32. Goldaracena N, Gorgen A, Doyle A, Hansen BE, Tomiyama K, Zhang W, et al. Live donor liver transplantation for patients with hepatocellular carcinoma offers increased survival vs. deceased donation. J Hepatol. 2019;70(4):666-673.

33. Duvoux C, Roudot-Thoraval F, Decaens T, Pessione F, Badran H, Piardi T, et al. Liver transplantation for hepatocellular carcinoma: a model including alpha-fetoprotein improves the performance of Milan criteria. Gastroenterology. 2012;143(4):986-94.e3; quiz e14-5.

34. Guichard C, Amaddeo G, Imbeaud S, Ladeiro Y, Pelletier L, Maad IB, et al. Integrated analysis of somatic mutations and focal copynumber changes identifies key genes and pathways in hepatocellular carcinoma. Nat Genet. 2012;44(6):694-8.

35. Thomas MB, Jaffe D, Choti MM, Belghiti J, Curley S, Fong Y, et al. Hepatocellular carcinoma: consensus recommendations of the National Cancer Institute Clinical Trials Planning Meeting. J Clin Oncol. 2010;28(25):3994-4005.

36. Vilgrain V, Pereira H, Assenat E, Guiu B, Ilonca AD, Pageaux GP, et al. Efficacy and safety of selective internal radiotherapy with yttrium90 resin microspheres compared with sorafenib in locally advanced and inoperable hepatocellular carcinoma (SARAH): an open-label randomised controlled phase 3 trial. Lancet Oncol. 2017;18(12): 1624-1636.

37. Nasralla DC, Coussios CC, Mergental H, Zeeshan Akhtar M, Butler AJ, Ceresa CDL, et al. A randomized trial of normothermic preservation in liver transplantation. Nature. 2018;557(7703):50-56

38. Miñambres E, Suberviola B, Dominguez-Gil B, Rodrigo E, Ruiz-San Millan JC, Rodríguez-San Juan JC, et al. Improving the Outcomes of Organs Obtained from Controlled Donation After Circulatory Death Donors Using Abdomnal Normothermic Regional Perfusion. Am J Transplant. 2017:17(8):2165-2172.

39. Line PD, Hagness M, Elnaes Berstad A, Foss A, Dueland S. A Novel Concept for Partial Liver Transplantation in Nonresectable Colorectal Liver Metastases: The RAPID Concept. Ann Surg. 2015;262(1):e5-9.

40. Pedica F, Ruzzenente A, Bagante F, Capelli P, Cataldo I, Pedron S, et al. A re-emerging marker for prognosis in hepatocellular carcinoma: the add-value of fishing c-myc gene for early relapse. PLoS One. 2013;8(7):e68203. 